\title{
Julia MAÑERO-CONTRERAS
}

Universidad de Sevilla. España. julmancon@alum.us.es

\author{
Estudio de caso de los sMOOC y su pedagogía en el contexto online
}

Case Study of sMOOC and its Pedagogy in the online context

\section{Resumen}

En un contexto dominado por las nuevas tecnologías, los MOOCs parecen posicionarse como una alternativa o complemento a la formación tradicional. Se observa sin embargo, que la metodología empleada por este tipo de cursos masivos, no difiere de la comúnmente utilizada en el aula. Este artículo evalúa y describe una nueva tipología de MOOC, denominada Social MOOC, la cual se presenta como disruptiva en cuanto a pedagogía se refiere. A través de la plataforma ECO Learning, se analiza de forma exhaustiva mediante un estudio de caso, la pedagogía empleada y el rol que asume el estudiante en el primer sMOOC llevado a cabo que tuvo lugar durante los meses de marzo y abril de 2015. Se concluye, que la pedagogía empleada en esta nueva versión social de los ya citados MOOC, es innovadora tanto dentro como fuera del contexto digital. Los estudiantes asumen un papel muy activo y la comunicación se torna de un carácter bidireccional entre el facilitador y los mismos.

\section{Palabras clave}

Educación; Curso en Línea; Formación Virtual; MOOC; Pedagogía; Social MOOC; Education; MOOC; Online Course; Pedagogy; Social MOOC; Virtual Education
Fecha de recepción: 30/04/2015 Fecha de revisión: 02/06/2016

Fecha de preprint: 25/06/2016 Fecha de publicación final: 01/07/2016

\begin{abstract}
In a context dominated by new technologies, MOOCs seem to be an alternative or complement to traditional training. However, it is observed that methodology used by this type of massive courses, is not different from that one commonly used in a classroom. This article describes and evaluates a new type of $\mathrm{MOOC}$ called Social MOOC, which is presented as disruptive in a pedagogical way. Through the ECO Learning platform is analyzed exhaustively through a case study the pedagogy used and the role assumed by the student in the first sMOOC developed and which took place during the months of March and April in 2015. It is concluded that pedagogy employed in this new social version of $M O O C$ is innovative both inside and outside the digital context. Students take an active role and communication becomes a two-way process between the facilitator and pupils.
\end{abstract}

\section{Keywords}

Education; MOOC; Online Course; Pedagogy; Social MOOC; Virtual Education 


\section{Introducción}

Las nuevas tecnologías han demostrado desde sus comienzos, que poseen una gran influencia en cualquier ámbito de nuestra vida. Apareciendo como un hecho disruptivo, han revolucionado nuestro contexto social modificando la forma en la que nos relacionamos y nos comunicamos. Inmersas en un escenario dónde el conocimiento evoluciona de forma constante, han posibilitado lugares de creación de conocimiento común. En ellos, todos y cada uno de nosotros hacemos posible un intercambio de información constante, llevando a cabo un proceso de enseñanza-aprendizaje de forma ubicua (Kalantzis y Cope, 2009), siendo tan sólo necesario tener acceso a un dispositivo con conexión a Internet. Sobre dicho proceso, podríamos añadir que: "el aprendizaje es cada vez más informal a medida que las personas desarrollan redes complejas para ayudarse entre sí"(Sangrà y Wheeler, 2013: 110).

Uno de dichos lugares de creación común de conocimiento, son los Massive Open Online Course (conocidos por su acrónimo MOOC). Esta terminología surge en el año 2008 de la mano de Dave Cornier (Vázquez y López, 2014) para hacer referencia a un curso impartido por George Siemens y Stephen Downes en el que añadieron el carácter de masivo a los ya conocidos cursos abiertos y en línea y por tanto son una evolución de éstos (Sánchez-Vera et al. 2014 y Valverde, 2014).

Este tipo de cursos, cobra gran popularidad en los últimos años en contexto español, debido a la necesidad por parte de la población de realizar un aprendizaje a lo largo de toda la vida, al encontrarnos inmersos en la llamada Sociedad del Conocimiento, término planteado por Castells en el año 2003. Según la publicación de Cuaderno Red de Cátedras Telefónica en el año 2014, un 35\% de las universidades españolas ofertan al menos un MOOC, siendo 111 el número total de MOOC ofertados (Oliver et al. 2014). La evolución de estos datos es progresiva ya que en nuestra sociedad el conocimiento se erige como la mayor fuente de poder, llegando a suponer el activo de más valor del que podamos disponer. Debido a este motivo, resulta necesario una actualización continua ligada a un reciclaje de los supuestos generales que conocemos.

Tomando como referencia un metaanálisis sobre los MOOC en contexto español, en el cual se establece una revisión de la bibliografía encontrada durante el periodo 2013-2014, (Sangrà et al. 2015) pretendemos exponer las líneas de investigación sobre esta temática más desarrolladas hasta la fecha.

Sobre un total de 228 estudios analizados, podemos considerar como principal línea de actuación aquella que centra su análisis en las estrategias pedagógicas implementadas, representado un total de $36,84 \%$ de las investigaciones totales.

El segundo foco de más importancia, versa sobre varias líneas con porcentajes cercanos como serían; la motivación e implicación de los estudiantes matriculados en un MOOC (28,07\%), el papel de las redes sociales en el proceso de enseñanza-aprendizaje $(23,24 \%)$ o los objetivos de las instituciones (22,37\%).

A partir de los datos expuestos, se demanda un análisis más exhaustivo de acuerdo con la perspectiva pedagógica, ya que parece estar sometida a las limitaciones de la plataforma y no a la temática en cuestión (Raposo-Rivas etal. 2014). Como crítica a nivel pedagógico, Bartolomé y Steffens (2014) concluyen con que los MOOC no establecen un valor añadido en términos pedagógicos en comparación con otros cursos en línea.

Profundizando en esta última idea, es cierto que la educación y el uso que ésta realiza de los nuevos medios debería estar en sintonía con las nuevas habilidades demandadas y con las nuevas formas por las que adquirimos el saber. Entre dichas características demandados se encuentran los sabios digitales (Prensky, 2010), personas alfabetizadas en materia digital, capaces de interaccionar, modificar, compartir y reutilizar la información, porque es así como se forma el conocimiento hoy día, entre todos y para todos. Este término de sabios digitales, 
estaría en sintonía con el de prosumers (Zafra, 2013), que viene a referirse a aquellas personas que actúan tanto como productores como consumidores de información, y por tanto realizan las tareas enumerada anteriormente por Prensky (2010).

Por tanto, cabe reflexionar sobre qué sentido tiene seguir trasmitiendo los conocimientos como se ha venido haciendo en el aula tradicional, en un contexto novedoso y con infinitas posibilidades como es el contexto online o digital ya que: "si en la economía del conocimiento todo el mundo es creador o creadora hay que poder imaginar modelos donde ni se restrinja ni se privatice ese potencial inmanente"(Acero, 2012: 54). De forma paralela, Carlos Escaño sugiere que estas peculiaridades del contexto pueden o bien ser aprovechadas o bien obviadas negando sus posibilidades (Escaño, 2013).

Existen dos tipologías de MOOC bastante afianzadas; los C-MOOC nacidos en el conectivismo y los X-MOOC basados en ideales conductuales y cognitivos (Moe, 2014; Torres y Gago, 2014). No obstante, el surgimiento de los Social MOOC, también denominado en adelante sMOOC, nos hizo (re)plantearnos sus novedades y posibilidades a nivel pedagógico. Abogando por un aprendizaje ubicuo y con unos criterios diferenciales, parecen situarse lejos de los demás tipos de MOOC conocidos hasta la fecha. Se denominan así mismo "sociales" porque su aprendizaje está determinado por la participación y la interacción social (Osuna y Camarero, 2016).

Por lo anteriormente expuesto, la aparición de esta incipiente tipología de MOOC, nos hizo plantearnos como objetivo principal de este estudio meramente exploratorio, realizar una aproximación a los Social MOOC en comparación con los demás MOOC de carácter generalista. En consecución de nuestro objetivo, se realiza un estudio de caso del primer sMOOC realizado en contexto español, donde se analiza la pedagogía y el aprendizaje propuesto. Así mismo, se realiza un análisis para responder a la pregunta principal de cómo la pedagogía propuesta favorece la creación de conocimiento común entre los propios estudiantes.

Tras esta observación, cabe responder a una segunda pregunta; si realmente se lleva a cabo una pedagogía innovadora o por el contrario sigue desarrollándose una pedagogía tradicional, como en lo que en un aula nos referimos. Produciéndose por tanto, una enseñanza conservadora desde el punto de vista metodológico, sólo que deslocalizada y que no atiende a conceptos como son, la capacidad crítica y creativa del capital humano, muy demandados en la sociedad actual.

\section{Metodología}

Considerándose los Social MOOC como un proyecto reciente y único, el estado del conocimiento del tema elegido es muy vago. Por ello, se podría definir la presente como una investigación de carácter exploratoria, con una finalidad descriptiva del fenómeno en cuestión. Siguiendo a Clares (2005), la metodología que se utiliza en este tipo de estudios es bastante flexible y amplia.

La metodología elegida dispuso de un enfoque mixto ya que, tras elegir como principal objeto de estudio un $\mathrm{SMOOC}$, se realizó un análisis de la experiencia tanto del investigador como de los propios estudiantes.

Poseemos por tanto un enfoque dual en nuestro estudio, que siguiendo a Corbetta (2007), la principal diferencia radica en que existe un enfoque deductivo, donde las teorías preceden a la observación (enfoque cuantitativo) frente a otro inductivo, de lo particular a lo general (enfoque cualitativo). Este hecho, lejos de suponer una limitación, nos confiere cierta riqueza ya que nos permite aproximarnos al objeto de estudio desde una perspectiva polifacética, que nos posibilita conocer la realidad social desde múltiples puntos de vista.

Para el análisis de la pedagogía propuesta desde los sMOOC, se formó parte del primer Social MOOC conocido hasta la fecha, ya que era un proyecto pionero. Dicha primera edición tuvo lugar entre los meses de marzo y abril de 2015.

39 
Realizando un síntesis del contexto, el denominado sMOOC: Paso a Paso fue llevado a cabo en la plataforma Eco Learning, una plataforma integrada en el proyecto ECO: Elearning, Comunicación y Open-data. Este proyecto es financiado por el CIP de la Comunidad Europea y cuenta con prestigiosas universidades y organizadores: UNED, Riverthia, Humance AG, European Association of Distance Teaching Universities, Instituto Superior de Ciências da Informaçao e Administraçao, Tabarca Consulting, Université Sorbonne Nouvelle, Fundación Universidad Loyola Andalucía, Sünne Eichler Beratung für Bildungsmanagement, Reimer IT Solutions B.V, University of Cantabria, University of Zaragoza, Politecnico di Milano, University of Oviedo, Universidade Aberta, BIC Euronova, University of Manchester, Universidad de Valladolid, Open University of the Netherlands, Geographica, Universidad de Quilmes y Universidad Manuela Beltrán.

Este proyecto, basado en los Recursos Educativos de Acceso Abierto (OER) y los MOOC, tiene como objetivo principal "ampliar el acceso a la educación y mejorar la calidad y el costoefectividad de la enseñanza y el aprendizaje en Europa" (Ecolearning, 2016).

Para el análisis del sMOOC nos servimos de una técnica interactiva, la observación participante. Cabe destacar la diferencia entre meramente observación y observación participante (Corbetta, 2007: 304), ya que, siguiendo la línea que se persigue desde los Social MOOC, se analizó que lo más productivo sería interaccionar con los demás participantes.

Mediante un estudio de caso, se evaluó sistemáticamente el sMOOC desde una óptica interna (como participante) y al mismo tiempo desde una óptica externa (como observador). Justificamos la elección de dicha técnica, debido a que a que se pretendía un mínima intrusión y no alterar el contexto donde se desarrollaba la acción, tratando de ser observadores en el contexto habitual y sin la intención principal de generalizar, sino de comprender el fenómeno en cuestión (Stake, 1999) y por tanto, un rol de completo participante, sin revelar nuestro misión de observación constante basándonos en Valles (1999).

Las actividades y hechos observados que resultaron pertinentes, se recogieron mediante un diario de campo, acompañando las anotaciones escritas con imágenes fotográficas realizadas a través de capturas de pantalla de la plataforma, existiendo por ende dos tipos de registros: el narrativo y el audiovisual.

En un segundo plano, y de forma complementaria, se realizó un cuestionario. Dicho cuestionario se llevó a cabo con miras a establecer futuras líneas de investigación en base a las impresiones de nuestro grupo de aprendizaje del Social MOOC. No se realizó ningún control sobre la muestra ya que se pidió participación voluntaria por parte del alumnado, siguiendo en cierta manera la dinámica de aprendizaje colaborativo llevada a cabo durante el sMOOC. Así pues, el número total de sujetos encuestados fueron 52, de los cuales 25 eran hombres y 27 mujeres. Tomando como referencia el número de participantes de nuestro grupo de trabajo en el sMOOC, que alcanzaba la cifra de 76 estudiantes la muestra analizada representa un 68\% de la población total.

La técnica de recogida de información, fue un cuestionario de elaboración propia formado por 10 preguntas, 9 de las cuales eran de estandarización cerrada y una de ellas abierta con el objetivo de recoger la opinión personal de los sujetos encuestados.

Lo ítems formaron una batería de preguntas y principalmente se establecieron preguntas de identificación, información y opinión sobre los MOOC y sus características. De forma paralela también se examinó la relación entre los MOOC y la creación/actualización del conocimiento.

El cuestionario fue validado por 4 expertos en materia de educación y nuevas tecnologías. Para dicha validación se tuvieron en cuenta dos aspectos fundamentales sobre los ítems propuestos:

1. Adecuación: correspondencia entre el contenido de cada pregunta y el nivel de preparación o desempeño del encuestado. 
2. Pertinencia: relación estrecha entre la pregunta, objetivos a lograr y el aspecto o parte del instrumento que se encuentra desarrollando.

Se valoraron ambos aspectos en una escala de 1 a 5, donde 1 fue la mínima adecuación o pertinencia y 5 la máxima. En los casos en los que algún ítem fue valorado con 3 puntos o menos, los expertos tuvieron que indicar que mejoras consideraban oportunas, sirviendo por tanto como una guía de actuación al investigador.

\section{Resultados}

\subsection{Diseño, desarrollo y contexto del sMOOC}

Se nos antoja de cierta relevancia comenzar este epígrafe arrojando los datos obtenidos sobre la plataforma ECO Learning. Esta plataforma, en comparación con otras como es el caso de Coursera, no dispone de una oferta extremadamente amplia de cursos. En nuestro caso, la temática gira en torno al ámbito educativo. En cambio, es subrayable el hecho, de que un mismo curso se oferte en varios idiomas, tanto en su versión original como en otros idiomas a los que ha sido adaptado.

El SMOOC: Paso a Paso, estaba conformado por varios grupos según el idioma. Para el idioma español existían 11 grupos. El grupo número 8 fue el que se nos asignó, ya que se realizaba un reparto equitativo de los estudiantes para no saturar los foros. Nuestro grupo por consiguiente constó de 76 participantes de diferentes nacionalidades, todas de habla hispana.

Fueron 30 los días a través de los cuáles se desarrollaron 18 lecciones teórico-prácticas. Estas pretendían introducir el concepto de sMOOC y sus enclaves de diseño y organización. En todas ellas, el carácter social, por el que se torna diferenciador este tipo de cursos, estuvo presente. La participación fue bastante masiva, tanto por parte de los facilitadores como de los alumnos. Desde el primer módulo hasta el último con el que concluyó el sMOOC, se desarrollaron actividades en el foro, la plataforma e incluso las redes sociales.

Muestra de ello fue el seguimiento de los alumnos de habla hispana a través de las redes sociales y la plataforma: Twitter alcanzó los 146 seguidores, Facebook alrededor de 415 seguidores y en la plataforma se iniciaron 11 debates. El debate con menor acogida tan sólo consiguió 32 respuestas, siendo uno en concreto el más popular, que alcanzó la cifra de 245 respuestas por parte de los estudiantes.

En cuanto a los módulos de aprendizaje, cabe destacar que todas las actividades propuestas favorecen la participación y el aprendizaje colaborativo. La dinámica es muy similar en todas ellas; en primer lugar se visiona un video o presentación con material de referencia y se pide a los alumnos que lleven a cabo una actividad individual que será evaluada a ciegas por dos compañeros. Hasta que el estudiante no evalúe al menos dos actividades de otros compañeros no obtendrá su puntuación. La ponderación de la nota se realiza en función a una serie de ítems que se proporcionaban para facilitar esta tarea evaluadora.

Aunque se establecen una serie de tareas obligatorias, indispensables para superar el curso, también se anima en cada unidad a realizar trabajo voluntario en el foro y redes sociales. Se pide aportación de información que pueda ser útil a través de la búsqueda o experiencias propias en determinados temas y así generar un conocimiento común. Este elemento es lo que caracteriza a este tipo de MOOC, la generación del saber colectivo a través de canales de participación. Así pues, como ya hemos expuesto con anterioridad, tuvo una gran acogida la didáctica propuesta e incluso algunos participantes hicieron hincapié en el hecho de no tener muy claro cómo participar, debido a que no entendían bien el funcionamiento de la plataforma.

Otro dato recogido como un aspecto negativo a mejorar, de cara a seguir afianzando este tipo de enseñanza, es el hecho de la saturación informativa. Varias veces se recoge en el diario de 
campo la palabra "ansiedad" por parte del alumnado. Este concepto se relaciona con la multitud de vías de comunicación y aprendizaje.

Poniendo el foco sobre los materiales utilizados para la creación de contenidos en este tipo de cursos, dicho materiales fueron seleccionados atendiendo al concepto de ubicuidad, al ser uno de los pilares fundamentales en esta tipología de MOOC. Los recursos estaban disponibles para ser descargados y consultados desde cualquier dispositivo, ya que a la plataforma en cuestión, se podía acceder desde ordenador, tablet o móvil, siendo únicamente indispensable la conexión a Internet. Aunque el curso finalizó el 30 de abril de 2015, varios meses después se podía seguir accediendo al mismo y, aún no estando tutorizado, estaba disponible el contenido ya publicado, tanto los contenidos de los módulos como la información compartida en el foro o las redes sociales.

El carácter abierto de este tipo de MOOC, se tangibilizó por ultimo en el hecho de que estuviera disponible en varios idiomas distintos: inglés, alemán, francés, español, italiano y portugués. Además habían sido generados atendiendo a los principios de libres y abiertos (principios básicos que todo MOOC debería atender si atendemos al significado de este acrónimo).

\subsection{Aprendizaje colaborativo: el grupo 8}

El estudio se completa con algunos datos sobre los participantes del grupo 8 de habla hispana donde realizamos parte de nuestra observación participante.

Aunque el $81 \%$ de los encuestados afirmaron poseer estudios universitarios difieren en los motivos por los que se decantaron en realizar el sMOOC. Las opciones más elegidas fueron aquella que hizo alusión a la flexibilidad del espacio y tiempo (36\%) y por la calidad de los contenidos propuestos (20\%) en segundo lugar.

El $76,9 \%$ de los alumnos, afirmaron haber realizado entre 1 y 3 MOOC durante el año 2014 y solo un $7,7 \%$ no había realizado ninguno, por tanto podían comparar su experiencia pasada con la actual. Para ello se les pidió que puntuaran varios aspectos del sMOOC realizado. Se les propuso en una escala del 1 al 5, que valoraran su experiencia donde 1 significaba negativa y 5 muy positiva siendo 3 neutra. Los ítems propuestos fueron por el siguiente orden: metodología, organización, calidad de los contenidos, recursos, motivación, multimedia y singularidad. Todos estos aspectos recogieron la mayor parte de las respuestas en la variable 4 (positiva). Realizando una comparativa, podemos apreciar como el concepto metodología fue el que obtuvo mayor número de respuestas positivas.

El $100 \%$ de los encuestados, estuvo de acuerdo en que un aprendizaje a lo largo de toda la vida es necesario. Cuándo de forma libre se les preguntó porqué creían que esa formación continua era necesaria, la mayoría citan la palabra "cambios" o "reciclaje" e incluso alguno se atreve afirmar que el aprendizaje continuo ha existido a lo largo de toda la modernidad.

Para la consecución de esta actualización del conocimiento, la mayor parte de los encuestados, concretamente un $92 \%$, estuvo de acuerdo en que a través de estos MOOC se genera un conocimiento nuevo.

\section{Discusión}

Con el fin de establecer una diferenciación entre los sMOOC como hito disruptivo, y los MOOC y por consiguiente, poder responder a nuestra pregunta de investigación planteada al comienzo de este estudio, se ha realizado una comparativa con los resultados obtenidos. De esta manera podremos concluir afirmando o refutando si los SMOOC introducen una pedagogía innovadora y diferencial con respecto a las demás tipología de MOOC 
Para ello, hemos establecido una serie de consideraciones en relación con un estudio publicado por Raposo-Rivas et al. (2014) en el que se analizaron 117 MOOC.

Los SMOOC difieren en la mayoría de los conceptos que ellos plantean, en relación con el perfil de MOOC que existe en habla hispana y por lo tanto con sus componentes pedagógicos. La comparativa entre los porcentajes recabados del estudio y los resultados de nuestra observación se presenta a continuación.

Aunque en más de la mitad de los $\mathrm{MOOC}(53,8 \%)$, no se especifica hacia a quién va dirigido el curso, esa información sí se encuentra en la presentación del sMOOC, especificando que va claramente dirigido a docentes o especialistas en la materia.

La interactividad, valor diferenciador de los SMOOC, apenas está presente en los cursos ofertados en el resto de plataformas ya que "las plataformas condicionan los diseños pedagógicos de los MOOC" (Raposo-Rivas et al. 2014, 34). Incluso otros autores, como Hew y Cheung (2014), hablan de la falta de interacción en los MOOC como barreras a superar.

Los MOOC promovidos por iniciativa particular, son inexistentes prácticamente $(11,1 \%)$, hecho relevante ya que, los estudiantes que realizaron sMOOC: Paso a Paso, tuvieron la posibilidad de crear su propio sMOOC poniendo en práctica aquellos conocimientos adquiridos.

Los objetivos del curso y de los módulos de aprendizaje en el sMOOC, siempre han estado disponibles para el alumnado, considerando de vital importancia la toma de consciencia de los objetivos a conseguir y para que puedan servir de guía para el aprendizaje del alumnado. No sucede lo mismo en la mayor parte de los cursos analizados en el estudio de Raposo- Rivas, ya que el $57,3 \%$ de los cursos omiten este apartado y nos hace plantearnos, cómo se puede establecer un aprendizaje autodirigido desde los $\mathrm{MOOC}$, sin que los propios estudiantes tengan claras las metas y los objetivos que se proponen alcanzar.

No obstante, considerando otro apartado fundamental de los sMOOC, como es su carácter social y por consiguiente la generación de conocimiento, podemos observar como los sMOOC difieren de la mayor parte de MOOC y es que: "una de las principales características de los sMOOC es su intercreatividad" (Acedo y Cano $2016: 119)$. Esto es debido a que la mayoría de MOOC, están diseñados para llevar a cabo actividades que buscan su resolución de una forma individual y no se hace necesaria la interacción (Chiappe et al. 2015). Este es un aspecto que claramente va en relación con la postura de Poy y Gonzales-Aguilar (2014), que consideran que se debe avanzar con respecto a la metodología tradicional en el aula.

Estando el sMOOC diseñado para realizar actividades de tipo colaborativas, en grupo y de forma individual algunas, se contrapone de nuevo con la mayoría de los MOOC. Así pues, en un estudio dónde se analizan $87 \mathrm{MOOC}$ y su forma de evaluar, se encuentra un alto porcentaje de cursos que se evalúan de forma automática en la plataforma o simplemente por pares (Gallego et al. 2015: 91).

En referencia a la visión de los propios estudiantes, estos respondieron de forma activa a una reflexión planteada en el foro, dónde se les preguntaba cómo debería ser un MOOC para facilitar el conocimiento común. Todos los estudiantes que respondieron a la cuestión estaban de acuerdo en que en el caso concreto de los sMOOC, se fomenta más la colaboración y la participación que en otro tipo de MOOC que hubieran realizado con anterioridad. Por tanto se pone de manifiesto que valoran la pedagogía desarrollada en comparación con las anteriores y que son conscientes de la diferencia en lo que a interactividad nos referimos y es que "el aprendizaje a lo largo de toda la vida se ha vuelto un aspecto importante sobre cómo aprendemos y dónde"(de Freitas et al. 2015: 455). 


\section{Conclusiones}

Podríamos culminar nuestro estudio, afirmando que los Social MOOC sí presentan una pedagogía innovadora con respecto a las demás tipologías de MOOC. Del trabajo realizado se desprende que la pedagogía de los mismos se encuentra determinada por varias conceptos desarrollados a continuación: el carácter social, la generación de conocimiento común y la comunicación bidireccional entre facilitadores y estudiantes.

El estudio realizado ha puesto de manifiesto que el carácter social de los $\$ M O O C$ es la principal diferencia con otros tipos de MOOC como es el caso de los X-MOOC (conductista) O C-MOOC (conectivista) en el que la pedagogía empleada se asemeja más a una tradicional, donde el estudiante realiza un aprendizaje de tipo individual.

Es muy probable que la pedagogía que el sMOOC emplea, se adapte en gran medida al contexto y a la cultura social actual. El rol participante que la mayoría de nosotros adoptamos en la web 2.0 y las redes sociales, se hace visible también en el sMOOC ya que la participación y el aprendizaje colaborativo son los ejes fundamentales de esta tipología emergente.

Atendiendo a una de las preguntas de investigación, el sMOOC igualmente facilita el conocimiento común, ya que son numerosas las vías de participación, no sólo en la propia plataforma (con el foro y el microblog) sino en las redes sociales como Facebook y Twitter.

Otro de los motivos que explicarían este hecho es que tras el pertinente análisis de la pedagogía, pudimos afirmar que debido a la propuesta metodológica, formas de evaluar y canales de participación, la creación de conocimiento común se ve favorecida por este tipo particular de MOOC. Así mismo, el hecho de que los materiales estuvieran optimizados para ser visualizados desde diferentes dispositivos potencia ese conocimiento colaborativo.

El carácter colaborativo es esencial, al igual que una comunicación bidireccional. El hecho de que en los numerosos canales de participación, las interacciones sean prácticamente realizadas por estudiantes, planteándose dudas y reflexiones y que la evaluación sea realizada por compañeros, hacen que la figura del estudiante se convierta en un ente activo y esencial para llegar a cabo este tipo de pedagogía innovadora.

Hay que hacer notar que en los sMOOC el estudiante tiene una responsabilidad, ya que tanto aprende como enseña. Existe una comunicación bidireccional entre los estudiantes y los facilitadores de grupo. El alumno se convierte en un ente activo y decide sobre su propio aprendizaje.

Los sMOOC nos han permitido conocer, las posibilidades que ofrece este nuevo tipo de educación basado en contexto de educación digital. Siendo el uso apropiado de las nueva tecnologías para el aprendizaje, una oportunidad al alcance de todos, si se analiza de forma consecuente como potenciar las nuevas formas de enseñanza. Por tanto, el gran impacto investigativo que genera este fenómeno, debería centrarse en la forma de obtener el máximo beneficio para el avance de una educación de todos y para todos.

\section{Referencias bibliográficas}

[1] ACERP, F. (2012). Cultura libre digital. Barcelona: Icaria.

[2] BARTOLOMÉ, A.; STEFFANS, K. (2014). ¿̇Son los MOOC una alternativa de aprendizaje? Comunicar, 21 (44), 91-99. https://www.doi.org/10.3916/C44-2015-10ertfgf

[3] CASTELLS, M. (2003). La era de la información: Economía, Sociedad y Cultura. Vol. 1 La sociedad red. Madrid: Allianza Editorial.

[4] CHIAPPE, A.; HINE, N. Y MARTíNEZ-SILVA, J-A. (2015). Literatura y práctica: una revisión crítica acerca de los MOOC. Comunicar, 21 (44), 09-18. https://www.doi.org/10.3916/C44-2015-01 
[5] CLARES, J. (2005). Informática aplicada a la investigación cualitativa. Sevilla. Universidad de Sevilla.

[6] CORBETTA, P. (2007). Metodología y técnicas de investigación social. Madrid: McGrawHIII/Interamericana.

[7] ECOLEARNING (2016). Ecolearning. Disponible en http://project.ecolearning.eu/es/

[8] ESCAÑO, C. (2013). Educación move commons. Procomún, cultura Libre y acción colaborativa desde una pedagogía crítica, mediática y e-visual. Arte, Individuo y Sociedad, 25 (2), 319-336. https://www.doi.org/10.5209/rev_ARIS.2013.v25.n2.39078

[9] DE FREITAS, S.; MORGAN, J. Y GIBSON, D. (2015). Will MOOCs transform learning and teaching in higher education? Engagement and course retention in online learning provision. British Journal of Educational Technology, 46(3), 455-471.

https://www.doi.org/10.1111/bjet.12268

[10] GALLEGO, M. J.; GÁMIZ, V. Y GUTIÉRREZ, E. (2015). Tendencias en la evaluación del aprendizaje en cursos en línea masivos y abiertos. Educación XX1, 18(2), 77-96. https://www.doi.org/10.5944/educXX1.12935

[1 1] HEW, K. F.; CHEUNG, W. S. (2014). Students' and instructors' use of massive open online courses (MOOCs): Motivations and challenges. Educational Research Review, (12), 45-58. https://www.doi.org/10.1016/j.edurev.2014.05.001

[12] KALANTZIS, M.; COPE, B. (2009). Ubiquitous Learning. Disponible en http://goo.gl/7OvinR

[13] OLIVER, M. et al. (2014). MOOCs en España. Cuadernos Red Cátedras de Telefónica. Disponible en http://goo.gl/DqNVBF

[14] OSUNA, S. Y CAMARERO, L. (2016). The ECO european project: A new MOOC dimension based on an intercreativity environment. Turkish Online Journal of Educational Technology, 15(1), 117-125. Disponible en http://goo.gl/lj49Jh

[15] POY, R.; GONZALES-AGUILAR, A. (2014). Factores de éxito de los MOOC: algunas consideraciones críticas. Iberian Journal of Information Systems and Technologies, 1 (e 1), 105-

118. Disponible en http://goo.gl/locLJu

[16] PRENSKY, M. (2010). Homo sapiens digital: de los inmigrantes y nativos digitales a la sabiduría digital. Disponible en http://goo.gl/qvPzTr

[17] RAPOSO-RIVAS, M.; MARTíNEZ-FIGUEIRA, E. Y SARMIENTO, J. A. (2014). Un estudio sobre los componentes pedagógicos de los cursos online masivos. Comunicar, 21 (44), 27-35. Disponible en https://www.doi.org/10.3916/C44-2015-03

[18] ROLIN, M. (2014). The evolution and impact of the Massive Open Online Course (Tesis Doctoral). Pepperdine University, California.

[19] SÁNCHEZ-VERA, M.; LEÓN-URRUTIA, M. Y DAVIS, H. (2014). Desafíos en la creación, desarrollo e implementación de los MOOC: El curso de Web Science en la Universidad de Southampton. Comunicar, 21 (44), 37-44. Disponible en http://goo.gl/muEoOG.

https://www.doi.org/10.3916/C44-2015-04

[20] SANGRÀ, A.; GONZÁLEZ-SANMAMED, M. Y ANDERSON, T. (2015). Metaanálisis de la investigación sobre mooc en el período 2013-2014. Educación XX1,18(2), 21-49. Disponible en http://goo.gl/tgyCf4. https://www.doi.org/10.5944/educxx1.13463

[21] SANGRÀ, A. Y WHEELER, S. (2013). Nuevas formas de aprendizaje informales: $\dot{\imath} O$ estamos formalizando lo informal? RUSC. Revista de Universidad y Sociedad del Conocimiento, 10 (1), 286-293. Disponible en https://goo.gl/5DF69N. https://www.doi.org/10.7238/rusc.v10i1.1689

[22] STAKE, R. (1999). Investigación con estudio de casos. Madrid: Morata. 
[23] TORRES, D.; GAGO, D. (2014). Los moocs y su papel en la creación de comunidades de aprendizaje y participación. RIED. Revista Iberoamericana de Educación a Distancia, 17(1), 1334. Disponible en http://goo.gl/763QNL. https://www.doi.org/10.5944/ried.17.1.11570

[24] VALLES, M. (1999). Técnicas Cualitativas de Investigación Social. Madrid: Sintesis Sociología.

[25] VALVERDE, J. (2014). Moocs: una visión crítica desde las ciencias de la educación. Profesorado, 18(1), 93-111. Disponible en http://goo.gl/Efjvc9

[26] VÁZQUEZ, E.; LÓPEZ, E. (2014). LOS MOOC y la educación superior: la expansión del conocimiento. Profesorado, 18(1), 3-12. Disponible en http://goo.gl/tWOIUY

[27] ZAFRA, R. (2013). (H)adas. Mujeres que crean, programan, prosumen, teclean. Madrid: Páginas de Espuma. 